\title{
Les Cent-Jours vus de la littérature, suivi d'un inédit de Charles de Rémusat, sous la direction de Bernard GENDREL et Mireille LABOURET
}

\section{Jacob Lachat}

\section{OpenEdition}

\section{Journals}

Édition électronique

URL : http://journals.openedition.org/studifrancesi/16428

DOI : $10.4000 /$ studifrancesi. 16428

ISSN : 2427-5856

\section{Éditeur}

Rosenberg \& Sellier

\section{Édition imprimée}

Date de publication : 1 juillet 2019

Pagination : 163-165

ISSN : 0039-2944

Référence électronique

Jacob Lachat, «Les Cent-Jours vus de la littérature, suivi d'un inédit de Charles de Rémusat, sous la direction de bernard Gendrel et Mireille LABouret », Studi Francesi [En ligne], 187 (LXIII | I) | 2019, mis en ligne le 01 juillet 2019, consulté le 25 janvier 2021. URL : http://journals.openedition.org/studifrancesi/ 16428 ; DOI : https://doi.org/10.4000/studifrancesi. 16428

Ce document a été généré automatiquement le 25 janvier 2021.

\section{(c) (i) (2) $\Theta$}

Studi Francesi è distribuita con Licenza Creative Commons Attribuzione - Non commerciale - Non opere derivate 4.0 Internazionale. 


\section{Les Cent-Jours vus de la littérature, suivi d'un inédit de Charles de Rémusat, sous la direction de Bernard GENDREL et Mireille LABOURET}

Jacob Lachat

\section{RÉFÉRENCE}

Les Cent-Jours vus de la littérature, suivi d'un inédit de Charles de Rémusat, sous la direction de Bernard GENDREL et Mireille LABOURET, Presses Universitaires de Strasbourg, 2017, $194 \mathrm{pp}$.

On voit se multiplier depuis quelques années des recherches consacrées au traitement des événements marquants $\mathrm{du} \mathrm{XIX}^{\mathrm{e}}$ siècle par la littérature. Qu'il s'agisse de la Révolution de 1789, de Waterloo, de la Révolution de Juillet, de Juin 1848 ou de la Commune, ces recherches s'intéressent à la façon dont les œuvres littéraires s'emparent d'objets largement balisés par l'historiographie, pour en offrir une vision singulière, voire une version décalée. Les Cent-Jours vus de la littérature s'inscrit dans cette actualité. Issu d'un colloque tenu en octobre 2015, cet ouvrage collectif a pour ambition d'étudier le regard qu'historiens, mémorialistes, romanciers et dramaturges ont porté sur les Cent-Jours, ce bref épisode qu'on considère généralement comme le point d'orgue de l'Empire, entre le retour fulgurant de Napoléon de l'île d'Elbe et sa chute dramatique à Waterloo. On connaît les dates - le $1^{\text {er }}$ mars et le 18 juin 1815 - qui bornent, pour reprendre les termes d'Alexandre Dumas, «cette courte apparition impériale». On connaît moins, en revanche, la variété des enjeux littéraires qui soustendent la représentation que nous nous faisons de cet intervalle historique. Car les Cent-Jours, au-delà de leur réalité politique, n'ont cessé d'intriguer les écrivains des $\mathrm{XIX}^{\mathrm{e}}$ et $\mathrm{XX}^{\mathrm{e}}$ siècles. Le volume vise précisément à étudier ce phénomène en élargissant 
l'empan chronologique au sein duquel il est souvent appréhendé. Il s'agit de comprendre la place paradoxale qu'occupent les Cent-Jours dans l'histoire littéraire. Comme le signalent B. Gendrel et M. Labouret dans leur introduction, bien que les CentJours soient un épisode connu, ils «forment un creux, un trou, une béance dans la représentation littéraire» (p. 8). Deux cents ans après les faits, tout l'enjeu consiste donc à relire des auteurs célèbres (Constant, Stendhal, Chateaubriand, Michelet, Hugo, Aragon, Anouilh, etc.) ou moins connus (Norvins, Thiers, Rémusat, Erckmann-Chatrian, etc.), afin d'examiner les "principales catégories esthétiques convoquées par [ces] auteurs» (p. 10) pour décrire ces trois mois d'exception dans l'histoire du XIX ${ }^{\mathrm{e}}$ siècle français.

2 La première question qui se pose aux chercheurs est celle du statut historique du phénomène en question. Faut-il envisager les Cent-Jours comme un événement ou comme une période? Faut-il les identifier comme un épisode clairement daté ou comme un moment de plus large ampleur, dont les conditions de possibilité et les conséquences débordent le cadre temporel de trois mois? Pour Natalie PETITEAU, spécialiste de l'histoire napoléonienne, il convient de contextualiser les Cent-Jours pas à pas, en prêtant attention aux différentes temporalités qui les traversent: "Ce court moment [...] est marqué à la fois par une incroyable accélération du temps et par d'étonnants retours en arrière» (p.15). Les autres articles du volume partent d'un constat similaire, mais en s'intéressant davantage à la façon dont ces temporalités sont pensées dans l'écriture de l'histoire. Ils montrent que, si les écrivains sont attentifs aux sursauts politiques et aux catastrophes militaires, ils demeurent souvent indécis quant à la consistance historico-politique de cette période partagée entre l'idée d'un retour du passé et celle d'un nouveau départ, soit entre une "puissance d'avenir» et un «futur antérieur» (p. 110), pour reprendre les termes de Franck LAURENT, qui explore les CentJours dans l'œuvre d'Hugo à travers la thématique du «retour». En effet, les Cent-Jours constituent autant un tournant politique qu'un bégaiement de l'histoire, et la plupart des textes qui racontent la période l'abordent de manière surprenante. Chez Stendhal, pourtant célèbre pour sa peinture de Waterloo dans La Chartreuse, les Cent-Jours sont étrangement relégués au second plan d'autres œuvres romanesques ou historiques, en particulier la Vie de Napoléon, où ils sont, d'après Marie PARMENTIER, «abordés sur le mode de l'anecdote, du détail: ils ne constituent pas l'objet ni l'enjeu du propos ou du récit, et l'épisode n'apparaît que de façon circonstancielle» (p. 85). Le même phénomène s'observe chez Balzac, qui, comme le montre Mireille LABOURET, évoque les Cent-Jours comme un «vide entre deux périodes également porteuses de sens et propices à l'action» (p. 76). D'autres écrivains s'interrogent de leur côté sur le sens qu'a pris cet épisode dans une histoire plus longue, au point d'en faire l'objet de méditations sur le principe de causalité. C'est notamment le cas de Dumas analysé par Julie ANSELminI: dans un essai intitulé Gaule et France (1833), l'écrivain «jauge [les CentJours et Waterloo] d'un point de vue téléologique, en fonction du sens qu'il assigne au mouvement de l'Histoire à travers les siècles» (p. 103). C'est aussi le cas des historiens du XIx ${ }^{e}$ siècle (Guizot, Thiers, Michelet, etc.) étudiés par Aude DÉRUELLE, qui font des Cent-Jours un «moment de bascule», tout en hésitant entre une «rationalisation de l'événement» en termes de "nécessité» et une «exploration d'autres devenirs historiques possibles en brèves uchronies» (p. 37). À croire que les écrits consacrés à cet épisode préfigurent les enjeux épistémologiques qui sont au cœur de recherches plus 
actuelles sur l'histoire contrefactuelle (pensons au récent ouvrage de Q. Deluermoz et P. Singaravélou, Pour une histoire des possibles, Paris, Editions du Seuil, 2016).

3 Toutefois, l'intérêt pour ces enjeux épistémologiques reste secondaire dans Les CentJours vus de la littérature. Le volume porte moins sur la question des possibles historiques ou des futurs non-advenus, que sur le destin politique et littéraire de Bonaparte, personnage principal de ce moment crucial. En se penchant sur des œuvres singulières, la plupart des articles analysent la fabrication ambivalente de la «légende impériale» (p.39). Malgré la variété des auteurs et des genres étudiés, leur préoccupation commune porte sur le traitement narratif de l'itinéraire de Napoléon depuis son débarquement à Golfe-Juan. Cet itinéraire a souvent été perçu comme un «véritable roman d'aventure» (p.15), et les événements qui le circonscrivent-le très métaphorique «Vol de l'Aigle» et l'étrange défaite de Waterloo - ont très tôt été racontés et interprétés à l'aune de modèles littéraires. Comme le montre brillamment Aude DÉRUELLE au sujet de l'historiographie romantique, les écrivains qui cherchent à marquer leur position face à Napoléon recourent volontiers au modèle théâtral: sous leur plume, l'empereur apparait tantôt en "héros tragique» (p.33), comme chez Lamartine ou Thiers, tantôt en «mauvais histrion» (p. 34), comme chez Chateaubriand ou Michelet; mais dans la plupart des cas, «le recours au modèle théâtral permet de [...] modéliser une lecture qui va toujours dans le sens d'une condamnation plus ou moins acerbe du retour de l'empereur, à ceci près que le modèle tragique lui confère une dignité que lui ôte brutalement le modèle comique» (p. 35). D'où la fonction critique de ce modèle qui, comme chez Marx, suppose une vision de l'histoire où les événements et les personnages se répètent tour à tour comme des tragédies et des farces. Comme l'explique Bernard GENDREL, des mémorialistes comme Chateaubriand ou Rémusat utilisent le modèle théâtral comme une «stratégie» propre à souligner leur distance face à la situation historique: cette distance tient à «l'inhérente ambivalence d'un tragique qui condamne le crime et sympathise avec lui, parce qu'il a de la grandeur, et d'un comique qui utilise alternativement rire bienveillant ou malveillant» (p. 62).

4 Parcouru de bout en bout par des remarques stimulantes sur ce genre de motifs littéraires dans l'écriture de l'histoire, le volume invite ainsi à redéployer la multiplicité des points de vue sur le retour de l'empereur dans une France «partagée en deux» (p. 18). Il suggère également que, dans le cas de l'histoire napoléonienne, l'usage de certains genres littéraires ne découle pas d'un simple souci esthétique, mais qu'il sert avant tout à appuyer le regard ironique que les écrivains portent sur les CentJours. Parmi ces genres littéraires, c'est le roman qui semble opérer «un pas de côté face à l'histoire en cette période où aucun parti ne semble bon» (p. 63). Les écrivains y recourent jusqu'au milieu du $\mathrm{xx}^{\mathrm{e}}$ siècle, mais dans une perspective bien différente de celle des romans historiques du XIX $\mathrm{X}^{\mathrm{e}}$. Par exemple, dans La Semaine sainte (1958), Aragon raconte l'épisode en ne mentionnant ni le vol de l'Aigle ni l'épopée de Waterloo: son roman, qui met en scène des "Cent-Jours sans Napoléon", paraît à une époque où, comme le rappelle justement Denis LABOURET, «la réflexion sur l'investigation de l'histoire par les moyens propres du roman est d'actualité» (p. 138).

5 C'est peut-être sur cette question de la spécificité des genres et de l'historicité des textes que le volume, considéré dans son ensemble, présente quelques faiblesses. Bien que les articles qui le composent soient répartis sur trois sections distinctes «Témoigner, reconstruire», «Regretter, magnifier», «Se souvenir, réinventer»-, qui correspondent grosso modo à une évolution chronologique, il est difficile d'appréhender 
sans justification la variété des écrits étudiés comme appartenant à un corpus cohérent. D'autant que la structure de l'ouvrage semble reconduire de manière ambiguë une dichotomie entre histoire et littérature que les contributeurs semblent pourtant chercher à dépasser: les articles à caractère historique ou historiographique ceux de Natalie PETITEAU (Les Cent-Jours devant l'histoire, pp. 15-26), d'Aude DÉRUELLE (Les Cent-Jours vus par l'historiographie romantique, pp. 27-38), d'Alex LASCAR (Norvins face aux Cent-Jours. Un admirateur embarrassé, p. 39-53) et de Bernard GENDREL (Les Cent-Jours ou la réécriture ambiguë de l'histoire (Constant, Chateaubriand, Rémusat), pp. 55-64) - sont rangés du côté du factuel («témoigner»), alors que ceux qui portent sur des œuvres tardives ceux de Gisèle SÉGINGER ("Waterloo" d'Erckmann-Chatrian: l'histoire racontée à hauteur d'homme, pp. 125-136), de Denis LABOURET (Les Cent-Jours, temps de l'aventure et "graines de l'avenir» selon Aragon, pp. 137-149) et d'Élisabeth LE CORRE (La "Foire d'empoigne" de Jean Anouilh: farce politique et mélodrame familial, pp. 151-164) - se situent du côté du fictionnel («réinventer»). Entre les deux pôles sont regroupés des articles sur des œuvres au statut générique parfois indécis - ceux de Mireille LABOURET (Les Cent-Jours vus d'un château de la Loire et d'autres lieux balzaciens, pp. 67-81), de Marie PARMENTIER (Les CentJours stendhaliens, "l'entreprise la plus romanesque et la plus belle des temps modernes», pp. 83-95), de Julie ANSELmini (Apparition de l'Empereur et vision de l'Histoire: les Cent-Jours vus par Dumas, pp. 97-107) et de Franck LAURENT (Un retour paradoxal: les Cent-Jours dans l'œuvre de Victor Hugo, pp. 109-121) -, puisque leurs auteurs ont eux-mêmes prétendu écrire l'histoire par les moyens du roman. Or, en intitulant leur volume Les Cent-Jours vus de la littérature, Bernand GENDREL et Mireille LABOURET n'indiquent nulle part ce que les lecteurs sont censés entendre, au juste, par «littérature ». S'agit-il de la mise en œuvre consciencieuse d'un récit historique ou de l'ensemble des procédés de fictionnalisation de l'histoire? Cette absence de définition, qu'elle découle d'un parti pris interprétatif ou d'un flottement théorique, prête en tout cas à confusion. À cette confusion vient s'ajouter un texte offert en annexe: un passage inédit des Mémoires de ma vie (1858) de Charles DE RÉMUSAT (p. 167-178). Inscrit tel quel et sans introduction au terme d'un ouvrage collectif consacré à la «littérature» sur un épisode historique, ce document bonus pourrait faire croire que les Cent-Jours, malgré la quantité d'archives qui s'y rapportent, n'ont été en définitive qu'un phénomène de papier. 